\title{
An Efficient DCT Compression Technique using Strassen's Matrix Multiplication Algorithm
}

\author{
Manish Manoria \\ Professor \& Director in CSE Department, TRUBA \\ Institute \\ of Engineering \&Information Technology, Bhopal, \\ India
}

\begin{abstract}
'Image compression' minimizes the problem that we face in storing and transmitting large amount of data. It reduces the size of data required to represent a digital image. In this procedure, DCT plays an important role. It separates information by using different frequencies. In Discrete Cosine Transformation (DCT), Quantization and encoding are the steps involved in the compression of the JPEG image. In this whole work, while using DCT, we have used Stassen's matrix multiplication algorithm for reducing the complex matrix multiplication problems. As per the result obtained from experiment, the performance of DCT is improved by using Stassen's matrix multiplication algorithm. The performance analysis is carried out through Peak signal to noise ratio (PSNR), and the different compression ratio (CR) for the different images.
\end{abstract}

Keywords - compression, strassen's matrix multiplication, CR, DCT, JPEG, PSNR.

\section{INTRODUCTION}

The term Image compression is minimizes the size of bytes of a graphics file without degrading the quality of the image. The decrease in file size allows more images to be stored in a given amount of disk or memory space, it allows rapid growth of computer applications and fulfills our need for efficient ways of storing large amount of data. The large amount of space required for holding huge collection of images can be very large in terms of cost [1]. Advantageously, there are several methods of image compression available today. The JPEG process is one of them that widely used, form of lossy image compression that based on the Discrete Cosine Transform (DCT) that work as separating images into parts of differing frequencies. Using a step called quantization, where the actual part of compression occurs, where only the most important frequencies that remain are used to retrieve the image in the decompression process and the less important frequencies are discarded. As a result, reconstructed images contain some distortion but as we shall soon see, during the compression stage these levels of distortion can be adjusted. The JPEG method is used for both colour and black-and-white images [1], but the center will be on compression. The previously done work on this field is not too good in terms of performance. A lot of work is going on to improve the performance of DCT.

In our research we focused on finding an efficient method to improve the performance of DCT. In this work we use Stassen's multiplication matrix algorithm to improve the performance of DCT.

\author{
Priyanka Dixit \\ CSE Department, TRUBA Institute of Engineering \\ \& \\ Information Technology, Bhopal, India
}

\section{BRIEF LITERATURE SURVEY}

DCT was firstly proposed in the year 1974 but that time it was not used for image compression. In 1984 first time it was used for image compression [2]. It is having a superiority in de correlation and energy compaction properties, the joint ISO committee adopts the DCT in the JPEG international compression standard, which is also the first compression standard for the amount of jpeg images. There are three purposes for which the image compression technique is widely used: firstly, it greatly compresses the image for limitation of storage, secondly it reduces the amount of information for transmission over the network and lastly it removes redundant information that can be very useful in Pattern recognition [2]. In JPEG standard, there are two compression techniques were defined these are lossy compression and lossless compression. In which the lossy compression technique is based on DCT. High compression could be achieved by lossy compression. In addition the vision effect of the reconstruction image from the process of decoding is similar with that of original image now there are amount of extending processes coding methods based on the basic DCT sequence processing. The basic JPEG image encoding method was defined in JPEG standard is composed with three procedures. The first procedure is to image transform, quantization and then encoding.

Discrete Cosine Transform (DCT) is a technique that widely used in image compression for converting a signal into frequency components.

\section{PROBLEM FORMULATION}

\subsection{The DCT Equation}

The DCT transforms the data from the spatial domain to the frequency domain. The spatial domain shows the amplitude of the color.The frequency domain shows how quickly the amplitude of the color is changing from one pixel to the next in an image file.

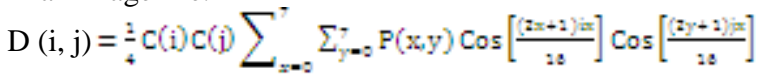

$$
\begin{aligned}
& C(u)= \begin{cases}\frac{1}{\sqrt{2}} & \text { if } u=0 \\
1 & \text { if } u>0\end{cases}
\end{aligned}
$$

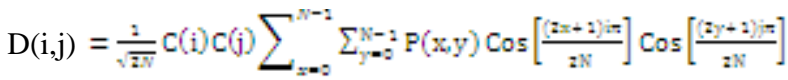


$P(x, y)$ is the $x$, yth element of the image represented by the matrix $P$. The equation calculates the term (i, j) of the transformed image from the pixel values of the original image matrix and $\mathrm{N}$ is the size of the block For the standard $8 \times 8$ block that uses the JPEG compression, $N$ equals 8 and $x$ and $y$ range from 0 to 7 . Therefore $D(i, j)$ would be as in following equation [1].The DCT uses cosine functions, the resulting matrix depends on the horizontal, diagonal, and vertical frequencies [1][2].

The image data is divided up into $8 \times 8$ blocks of pixels. A DCT is applied to each $8 \times 8$ block. DCT converts the spatial image representation into a frequency domain, the low-order represent by ' $\mathrm{AC}$ ' the average value in the block, while successive higher-order represent by 'DC' the strength of more rapid changes across the width or height of the block [1].

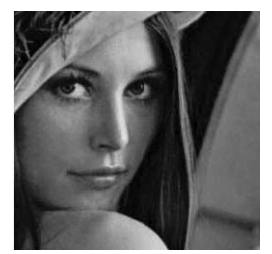

Lena image

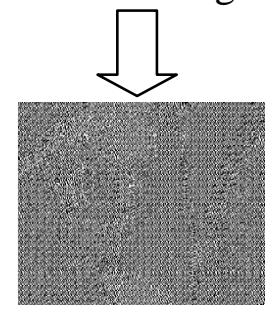

DCT image

\section{Figure.1 Block diagram of the JPEG Image Original Image} into DCT

\subsection{Quantization}

Quantization refers to the process of approximation of the continuous set of values in the image data with a finite set of values. The input values are the original data, and the output is always a finite number of levels. The quantizer is functions that have a set of output values are discrete, and generally finite. The quantization is done by basically dividing each component in the frequency domain by a constant for that component and then rounding to the nearest integer. It is one of the lossy operations in the whole compression process [1]. A remarkable and highly useful feature of JPEG process is that in this step, varying levels of image compression and quality are obtained through selection of specific quantization matrices[6]. This enables the user to decide on quality levels ranging from 1 to 100 , where 1 gives the poorest quality and the highest compression and 100 gives the best quality and lowest compression[1][12]. Quantization involves dividing each coefficient by an integer between 1 and 255 and rounding off [1]. JPEG standards give out a standard quantification matrix table, for the gray image, only need to brightness quantification table, as shown in table [4].
TABLE.1 BRIGHTNESS QUANTIZATION TABLE

\begin{tabular}{|l|l|l|l|l|l|l|l|}
\hline 16 & 11 & 10 & 16 & 24 & 40 & 51 & 61 \\
\hline 12 & 12 & 14 & 19 & 26 & 58 & 60 & 55 \\
\hline 14 & 13 & 16 & 24 & 40 & 57 & 69 & 56 \\
\hline 14 & 17 & 22 & 29 & 51 & 87 & 80 & 62 \\
\hline 18 & 22 & 37 & 56 & 68 & 109 & 103 & 77 \\
\hline 24 & 35 & 55 & 64 & 81 & 104 & 113 & 92 \\
\hline 49 & 64 & 78 & 87 & 103 & 121 & 120 & 101 \\
\hline 72 & 92 & 95 & 98 & 112 & 100 & 103 & 99 \\
\hline
\end{tabular}

\section{3 Zigzag method}

After quantization, the DC coefficient is treating separately from the $63 \mathrm{AC}$ coefficients [7]. The DC coefficient is a measure of the average value of the 64 image samples. Because there is usually strong correlation between the DC coefficients of adjacent $8 \times 8$ blocks, the quantized DC coefficient is encoded as the difference from the DC term of the previous block in the encoding order, (as shown in Figure 2)[1][2]. Zigzag method is done so that the coefficients are in order of increasing frequency. The higher frequency coefficients are more likely to be 0 after quantization. This improves the compression of run-length encoding.

\subsection{Run-length Coding}

The consecutive zeros are grouped and the number of zeros in the group is encoded. This process is called "Run-Length encoding". The term Run means repeated occurrence of the same character. Number of repetition is called the length of the run.Run length encoding is applied to the AC coefficients

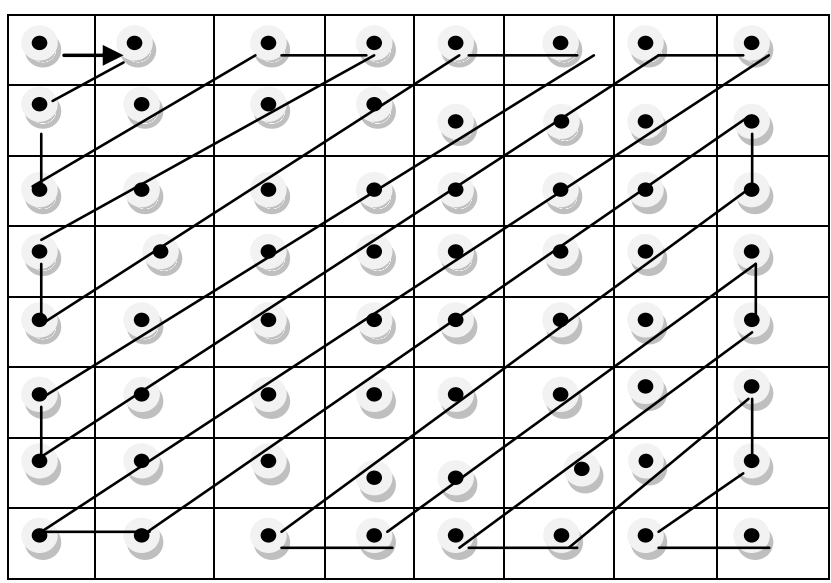

Figure.2 DCT Coefficient numbered according to Zigzag order.

of the matrix. Differential coding modulation is applied to the DC co-efficient. It allows a good way to incorporate a controlled loss in the encoding. Instead of reading across the rows, row after row, JPEG compression reads along the diagonals. This tends to groups the lower coefficients in the 


$$
\mathrm{B}=\left[\begin{array}{ll}
\mathrm{B} 11 & \mathrm{~B} 12 \\
\mathrm{~B} 21 & \mathrm{~B} 22
\end{array}\right]
$$

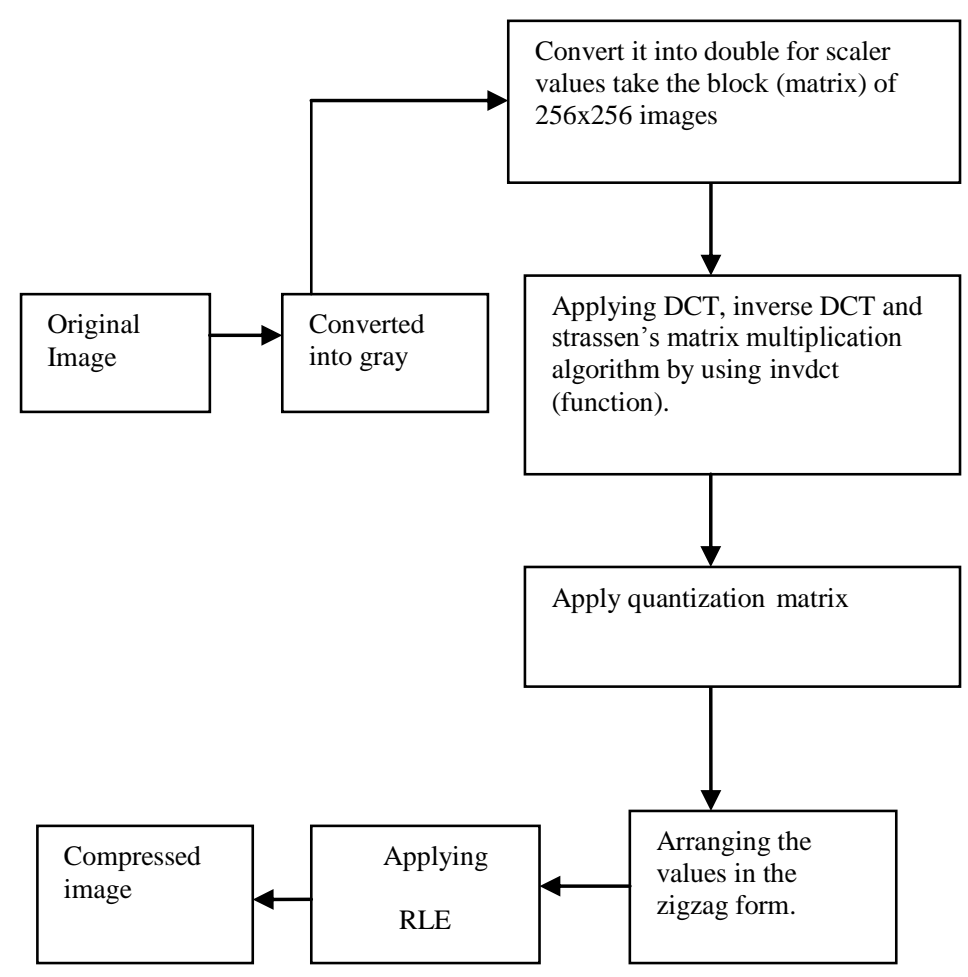

Figure.3 Block diagram of Algorithm used

beginning of the string and distributes the zeros in longer Continuous strings. To analyses the image compression algorithm using 2-dimension DCT. According to the DCT properties, a DC is transformed to discrete delta-function at zero frequency [5]. Hence, the transform image contains only the DC component. To transformed an image into $8 \times 8$ subsets by applying DCT in 2 dimensions. Also, a subset of DCT co-efficient has been prepared in order to perform inverse DCT to get the reconstructed image.

\section{STASSEN'S MATRIX MULTIPLICA- -TION ALGORITHM}

The standard method of matrix multiplication of two $n \times n$ matrices takes $\mathrm{O}\left(\mathrm{n}^{3}\right)$ operations. Stassen's algorithm is a Divide-and-Conquer algorithm that is asymptotically faster. The usual multiplication of two $2 \times 2$ matrices takes 8 multiplications and 4 additions. Strassen's shows how two $2 \times$ 2 matrices can be multiplied using only 7 multiplications and 18 additions. If we Imagine that $\mathrm{A}$ and $\mathrm{B}$ are each partitioned into four square Strassen showed that $2 \times 2$ matrix multiplication can be accomplished in 7 multiplication and 18 additions or subtractions. $\left(2_{2}^{\log _{2} 7}=2^{2.807}\right)$

\section{Stassen's algorithm}

1. Partition $\mathrm{A}, \mathrm{B}$ and and $\mathrm{C}$ into 4 equal parts:

$$
A=\left[\begin{array}{ll}
\text { A11 } & \text { A12 } \\
\text { A21 } & \text { A22 }
\end{array}\right]
$$

$$
\mathrm{C}=\left[\begin{array}{lll}
\mathrm{C} 11 & \mathrm{C} 12 \\
\mathrm{C} 21 & \mathrm{C} 22
\end{array}\right]
$$

2. Evaluate the intermediate matrices:

$$
\begin{aligned}
& \mathrm{M} 1=(\mathrm{A} 11+\mathrm{A} 22)(\mathrm{B} 11+\mathrm{B} 22) \\
& \mathrm{M} 2=(\mathrm{A} 21+\mathrm{A} 22) \mathrm{B} 11 \\
& \mathrm{M} 3=\mathrm{A} 11(\mathrm{~B} 12-\mathrm{B} 22) \\
& \mathrm{M} 4=\mathrm{A} 22(\mathrm{~B} 21-\mathrm{B} 11) \\
& \mathrm{M} 5=(\mathrm{A} 11+\mathrm{A} 12) \mathrm{B} 22 \\
& \mathrm{M} 6=(\mathrm{A} 21-\mathrm{A} 11)(\mathrm{B} 11+\mathrm{B} 12) \\
& \mathrm{M} 7=(\mathrm{A} 12-\mathrm{A} 22)(\mathrm{B} 21+\mathrm{B} 22)
\end{aligned}
$$

3. Construct $\mathrm{C}$ using the intermediate Matrices:

$$
\begin{aligned}
& \mathrm{C} 11=\mathrm{M} 1+\mathrm{M} 4-\mathrm{M} 5+\mathrm{M} 7 \\
& \mathrm{C} 12=\mathrm{M} 3+\mathrm{M} 5 \\
& \mathrm{C} 21=\mathrm{M} 2+\mathrm{M} 4 \\
& \mathrm{C} 22=\mathrm{M} 1-\mathrm{M} 2+\mathrm{M} 3+\mathrm{M} 6
\end{aligned}
$$

\section{Description of Algorithm:}

1. Partition $\mathrm{A}$ and $\mathrm{B}$ into quarter matrices as described above.

2. Compute the intermediate matrices: 
2.1 If the sizes of the matrices are greater than a threshold value, multiply the Recursively using Stassen's algorithm.

2.2 Else use the traditional matrix multiplicati- -on algorithm.

3. Construct $\mathrm{C}$ using the intermediate matrices.

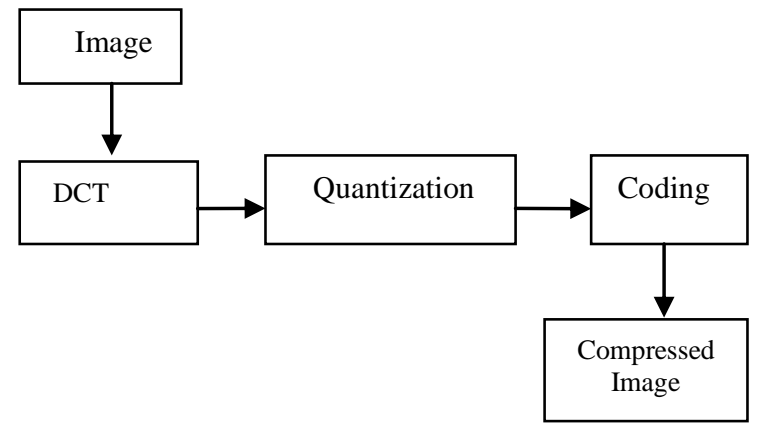

Figure.4 Block diagram of JPEG Image Compression process.

\section{PROPOSED METHODOLOGY}

The following is a general overview of the JPEG process.

The proposed algorithm is as mentioned:

1. The input image can be read by using imread function and then broken into $8 \times 8$ block of matrixes.

2. The algorithm can be applied for gray scale by suitably using functions such as is gray functions.

3. DCT is applied to each block on its both the rows and columns.Strassen's matrix multiplication algorithm is applied on the DCT matrix multiplication calculation.

4. Each block is compressed by quantization. Suitably the quantization matrix is selected. They are standard matrices used in JPEG.

5. The array of compression blocks that constitute the image is stored in a significantly reduced amount of space.

6. The image is reconstructed through decompression using Inverse DCT.

The following are the process of compression and decompression by using DCT image compression technique. In the (figure.4) [5] the process of compression can be shown and in the another (figure.5) [5] the process of decompression can be shown as for the decompression we use inverse DCT transformation technique

\section{EXPERIMENTAL RESULTS}

The jpeg compression based on DCT is favourable for reducing the overall processing time and the jpeg data based on compression domain is greatly reduced as compared to the original data, so it is very useful for improving the efficiency of the system. Some of the compression algorithm, to a certain extent, meets the requirement of the analysis and processing of the jpeg image data [4].DCT Compression technique can be applied to different JPEG images and different types of results

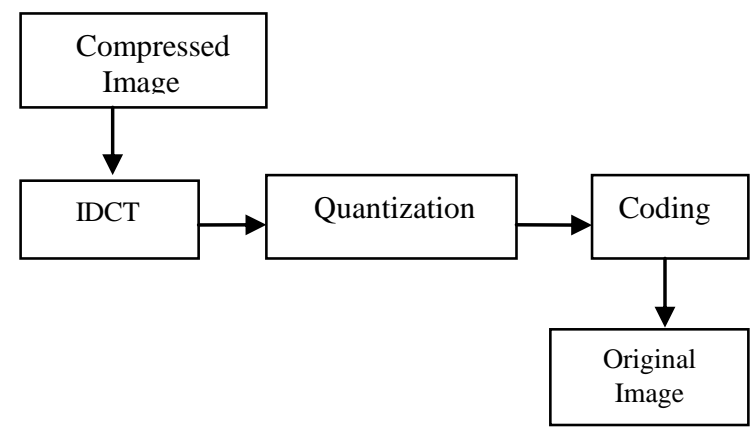

Figure.5 Block diagram of JPEG Image Decompression process.

can be obtained. We have performed several experiments to evaluate the performance of DCT on Jpeg image format using, MATLAB 7.5.

Hardware configuration of system on which all the experiments were conducted is- Intel Core 2 Duo processor and $100 \mathrm{Mbps}$ Internet
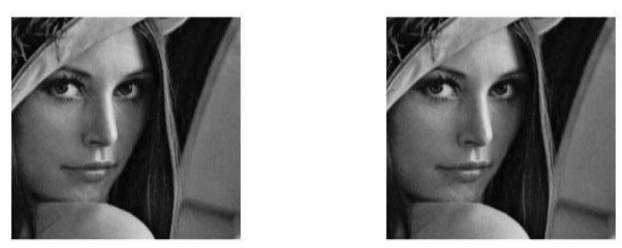

Original image

$8 \times 8$ lena.jpg
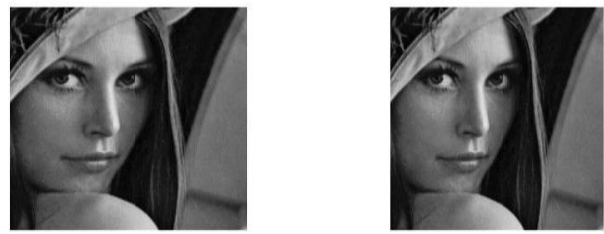

$32 \times 32$ lena.jpg

$128 \times 128$ lena.jpg

Image compression we use dct technique and the strassen's matrix multiplication algorithm to reduce the multiplication complexity. JPEG image compression and decompression is implemented using MATLAB. The various quantitative factors that are calculated are PSNR, and CR. 
Tabulation of results is carried out for 3 test images namely lena.jpeg, barb.jpeg and couple.jpeg. Compression factor is the factor by which the quantization matrix is multiplied. The performance can be calculated by some measuring parameters $\mathrm{CR}$, PSNR are measured. Compression factor decides the CR and hence the number of bits required to represent the compressed image[2].

The following figures the $\mathrm{X}$ axis indicates the compression ratio and $\mathrm{Y}$ axis indicates PSNR. Compression algorithm for three different images For different compression factors is shown in Figures 7, 8, 9 and 10[3].

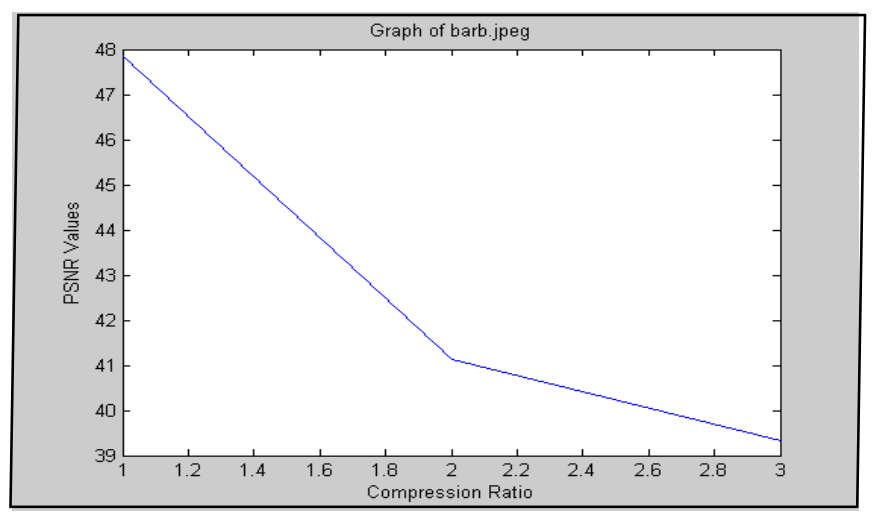

Figure.7 Result for image barb.jpeg

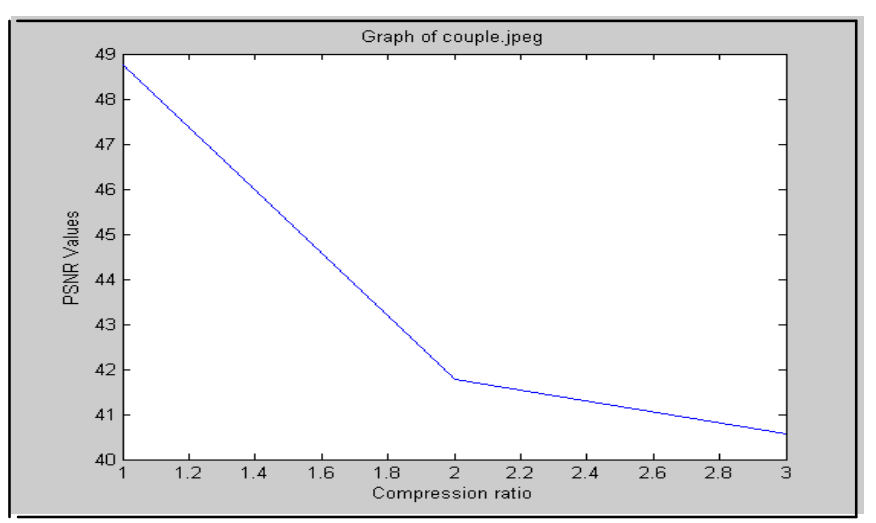

Figure.8 Result for image couple.jpeg

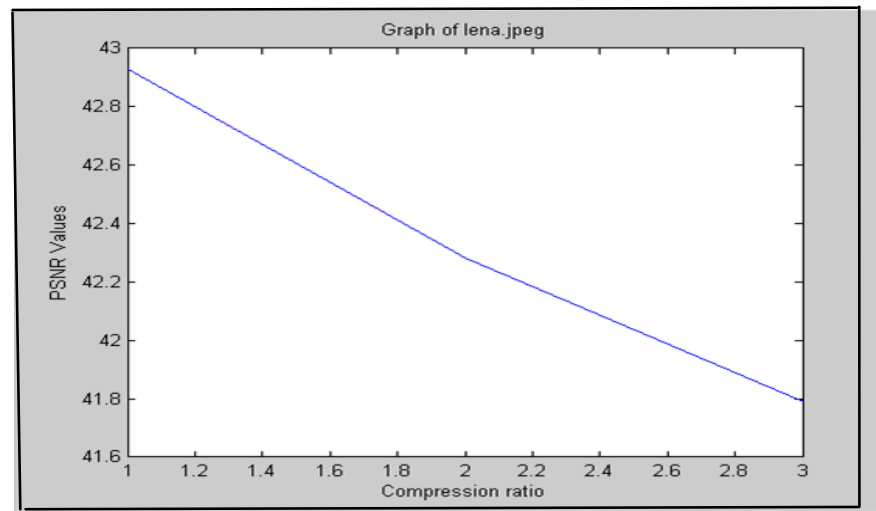

Figure.9 Result for image lena.jpeg

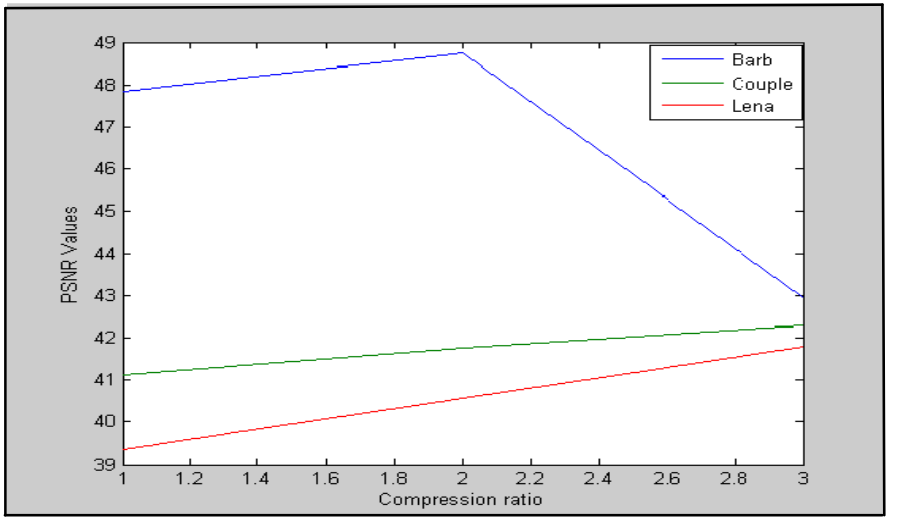

Figure 10. Result for all three images

For the different images even if the compression ratio remains same PSNR are found to be different. From these results it may be concluded that performance of JPEG will not remain same for the given compression factor $\&$ it changes from image to image [9]. The compressed data is widely used for the storage and transmission of the multimedia data. Therefore, image processing technology based on compressed domain can be applied in more areas. This technique of compression will provides the better results and performance.

\section{CONCLUSION}

The JPEG is a image compression standard. This paper analyzed main procedures of Image Compression which could be achieved using the procedure of DCT. The complexity of DCT is reduced, comparing with traditional algorithm. Furthermore, DCT is implemented based on the result redundant information with high frequency in an image could be removed by quantization or coding procedure. It means that DCT is the main foundation of compression in JPEG code of lossy compression. With the increase of compression ratio the more information could be lost. Therefore DCT algorithms contribute to high Encoding efficiency can hardly protect the resources of processors. The results presented in this document show that the DCT exploits interpixel redundancies to make excellent decorrelation for most natural images.

Table.2 Between the compression ratio / PSNR (DB)

\begin{tabular}{|c|c|c|c|}
\hline & \multicolumn{3}{|c|}{$8 \times 8$ DCT } \\
\hline Images & $8: 1$ & $32: 1$ & $128: 1$ \\
\hline Barb & 47.85 & 41.12 & 39.34 \\
\hline couple & 48.76 & 41.77 & 40.57 \\
\hline Lena & 42.93 & 42.28 & 41.79 \\
\hline
\end{tabular}


Thus, all (uncorrelated) transform coefficients can be encoded separately without compromising coding efficiency.

In addition, the DCT packs energy in the low frequency regions. Therefore, some of the high frequency content can be discarded without significant quality degradation. We use strassen's matrix multiplication algorithm to reduced the matrix multiplication complexity during the DCT process .Hence we get the remarkable performance of DCT during this method. The overall performance can be shown by some parameters like PSNR values of images after applying the proposed method on different images. Lastly, it is concluded that successive performance of DCT after applying Strassen's matrix algorithm for fast multiplication of complex matrix a compare with traditional methods.

\section{REFERENCES}

[1] S. V. Viraktamath et al.," Performance Analysis of JPEG Algorithm" IEEE, 978-1-61284-653-8/2011.

[2] Wei Zheng, et al" Research in a Fast DCT Algorithm Based on JPEG” IEEE, 978-1-61284-459-6/2011.

[3] Jingpeng LU et al "Survey on the Technology of Image Processing Basedon DCT Compressed Domain" IEEE, 978-1-61284-774-0/2011.

[4] Xi Hong Zhou et al," Research on DCT -based Image Compression Quality" 978-1-4244-9793-5/2011 IEEE.

[5] Zheng Wei et al," Analysis of JPEG Encoder for Image Compression" IEEE, 978-1-61284-774-0/2011.

[6] G. Valenzise, M. Tagliasacchi, S. Tubaro "The cost of JPEG compression anti-forensics" IEEE, 978-1-45770539-7/2011.
[7] Tiziano Bianchi, Alessandro Piva," Dete- ction of nonaligned double jpeg compression with estimation of primary compression param- eters" $18^{\text {th }}$ IEEE International Conference on Image Processing, 978-14577-1303-3/11/2011.

[8] Lin Ma, Student Member, Songnan Li, Student, Fan Zhang, and King Ngi Ngan," Reduced-Reference Image Quality Assessment Using Re organized DCTBased Image Representation"' IEEE, VOL. 13, NO. 4 , AUGUST 2011.

[9] Nabila Brahimi, Saad Bouguezel, "An Efficient Fast Integer DCT Transform for Images Compression with 16 Additions Only" 7th International Workshop on Systems, Signal Processing and their Applications (WOSSPA) -2011 .

[10] Aree Ali Mohammed, Jamal Ali Hussein, "Hybrid Transform Coding Scheme forMedical Image Application" 978-1-4244-9991-5/11/2011

[11] T.Sreenivasulu reddy, K.Ramani, S.Vara- --adarajan and B.C.Jinaga."Image Compressi- -on Using Transform Coding Methods", IJCS- NS International Journal of Computer Science and Network Security, VOL. 7 NO. 7.

[12] Ken Cabeen and Peter Gent, "image Compression and the Discrete Cosine Transform", Math 45, College of the Redwood.

[13] Mahendra M. Dixit, Prof. Priyatam kumar "Comparative Analysis of Variable Quantization DCT and Variable Rank Matrix SVD Algorithms for Image Compression Applications" 978-1-4244-5967$4 / 10 / 2010$

[14] F. M. Bayer and R. J. Cintra, "Image Compression via a Fast DCT Approximation" Latin America Transactions, VOL. 8, NO. 6, December 2010. 